\title{
Cambios en el régimen hídrico de la laguna Lasuntay y Chuspicocha por variaciones en el Nevado Huaytapallana
}

Changes in the hydric regime of the Lasuntay and Chuspicocha lagoons for variations in the snowfall Huaytapallana

Jacinto Arroyo Aliaga', Pedro Gurmendi Párraga ${ }^{2}$

Instituto Geofísico del Perú - Observatorio de Huancayo

\section{RESUMEN}

Objetivos: Estimar los efectos de las variaciones de torrentes de agua de los glaciares del Nevado Huaytapallana que emanan al sistema hídrico de las lagunas de Lasuntay y Chuspicocha. Métodos: Se utilizó el método general teórico deductivo de nivel explicativo, con un diseño no experimental del tipo transversal en el tratamiento de información; como método específico se ha utilizado el balance de masa del glaciar para el cálculo de volúmenes de agua de los torrentes a partir de la instalación de una red de balizas en la mayor parte del glaciar y en la zona de acumulación y ablación se excavaron pozos mediante perforaciones para medir directamente la cantidad de nieve acumulada entre el inicio y el fin del año hidrológico. Resultados: Se ha estimado los torrentes de caudal de agua que emanan del nevado Huaytapallana a las lagunas de Lasuntay y Chuspicocha en 1226700 m3 en la estación de verano; y de 245340 m3 en la estación de primavera. Conclusiones: El volumen máximo de acumulación en ambas lagunas fue en el verano y el volumen mínimo en la estación de primavera, debido a las variaciones en el régimen hídrico del sistema de acumulación y ablación que afectan la disponibilidad de agua.

Palabras clave: Régimen hídrico, variaciones, Lasuntay, Chuspicocha, Nevado Huaytapallana.

1 Investigador Adjunto en Prevención de Desastres y Cambio Climático del Instituto Geofísico del Perú.

2 Docente investigador de la Universidad Continental de Ciencias e Ingeniería. 


\section{ABSTRACT}

Objectives: Estimate effects of water torrents variations of snowfall Huaytapallana glaciers that come to the hydric system of the Lasuntay and Chuspicocha lagoons. Methods: There was used the deductive theoretical general method of exploratory level, with a non experimental design of cross-sectional type in the information treatment; as specific method it has been used the glacier mass balance to calculate water volumes of torrents starting with the installation of a beacons net on the glacier top and in the accumulation and ablation area were excavated water holes through by perforations to measure directly the snow accumulated quantity between the beginning and the end of the hydrological year. Results: There has been estimated that the volume of water torrents that come from the snowfall Huaytapallana, to the Lasuntay and Chuspicocha lagoons in $1226700 \mathrm{~m} 3$ in summer season; and $245340 \mathrm{~m} 3$ in spring season. Conclusions: The accumulation maximum volume in both lagoons was in summer and the minimum volume in the spring season because of variations in the hydric regime of the accumulation and ablation system that affects the water disposability.

Key words: Hydric regime, variations, Lasuntay, Chuspicocha, Huaytapallana glacier.

\section{INTRODUCCIÓN}

De todos los glaciares que se encuentran en los trópicos del mundo, la mayor superficie glaciar se halla en las montañas peruanas (1). Los glaciares del Perú cubren el 0,12\% de su superficie y concentra el $71 \%$ de los glaciares tropicales del mundo con 20 cordilleras identificadas con presencia de glaciares, y 3044 glaciares netos distribuidos en una superficie estimada de 1958 $\mathrm{km} 2$, todas ellas se encuentran en las cumbres de la cordillera de los andes, cuyo deshielo genera cerca del $98 \%$ de los recursos hídricos que escurren a la vertiente del Atlántico y menos del $2 \%$ a la vertiente del Pacífico (2). Los glaciares no son estáticos ni perpetuos. La formación de estas masas de hielo depende enteramente de las condiciones climáticas a escala regional y global (3). Se reporta que a partir de 1860, al término de la Pequeña Edad de Hielo, debido a alteraciones climáticas globales naturales, las montañas glaciares vienen sufriendo un proceso de desglaciación, acentuándose a partir de la década de 1980 (4).

En el Perú es visible el retroceso de los frentes glaciares hacía niveles más altos como consecuencia de una considerable pérdida de las masas de hielo; tanto así, que los glaciares pequeños con escasa $\circ$ ninguna zona de acumulación están desapareciendo (5). Los glaciares son reserva sólida de agua dulce del Perú. Sin embargo en los últimos 35 años se ha perdido el $22 \%$ de su superficie glaciar con referencia al año 1970 (6).

La situación problemática está referida a la necesidad de conocer los efectos de las variaciones de torrentes de agua de los glaciares del Nevado Huaytapallana que emanan al sistema hídrico de las lagunas de Lasuntay y Chuspicocha.

El objetivo principal es estimar los efectos de las variaciones de torrentes de agua de los glaciares del Nevado Huaytapallana que emanan al sistema hídrico de las lagunas de Lasuntay y Chuspicocha.

La investigación contribuye en el conocimiento de la variación del volumen máximo de acumulación en ambas lagunas en los diferentes ciclos estacionales. El verano que tiene características de fuertes precipitaciones sólidas es la fuente de acumulación neta en los glaciares. La primavera constituye la estación en dónde el estrés hídrico se agudiza por las pérdidas significativas en los volúmenes de agua acumulada. Sin embargo esta estación también se beneficia de los aportes netos de caudal de agua que emanan de la desglaciación. 
Queda como vacío metodológico la influencia real del sistema climático en la pérdida de masa glaciar en el tiempo. Así también queda dudas a una interrogante sobre ¿̇Por qué la isoterma tiene mayor variación en la vertiente occidental y menor en la vertiente oriental?

\section{MATERIAL Y MÉTODOS}

\section{Delimitación de la zona de estudio}

La zona de estudio fue en la vertiente occidental del nevado del Huaytapallana porque el agua que escurren de la fusión del nevado es la fuente de recarga hídrica de la laguna Lasuntay y Chuspicocha como se observa en la Figura $N^{0} 1$. Estas lagunas abastece de agua al río Shullcas, principal abastecedor de agua a la ciudad de Huancayo, capital de la región Junín.

\section{Estimación de torrentes de agua a partir del balance de masa glaciar:}

Esta medición se llevó a cabo a partir de perforaciones en la sección alta donde generalmente la acumulación supera la ablación para medir directamente la cantidad de nieve acumulada entre el inicio y el fin del año hidrológico para posteriormente convertir esta cantidad en su equivalente de agua ( $\mathrm{mm}$-equivalente). En la parte baja que escurre agua en forma de torrentes se midieron caudales que llegan a las lagunas, Figura $\mathrm{N}^{\circ} 2$ y 3.

También se utilizó el método de balance de masas de un glaciar para determinar el equivalente de agua ( $\mathrm{mm}$-equivalente) que gana y pierde en un tiempo determinado, mediante el cálculo de los cambios en la masa del glaciar y su distribución de dichos cambios en el tiempo y espacio. Esto nos permitió vincular una cadena de eventos de avances y retrocesos del sistema de almacenamiento de nieves. (8)

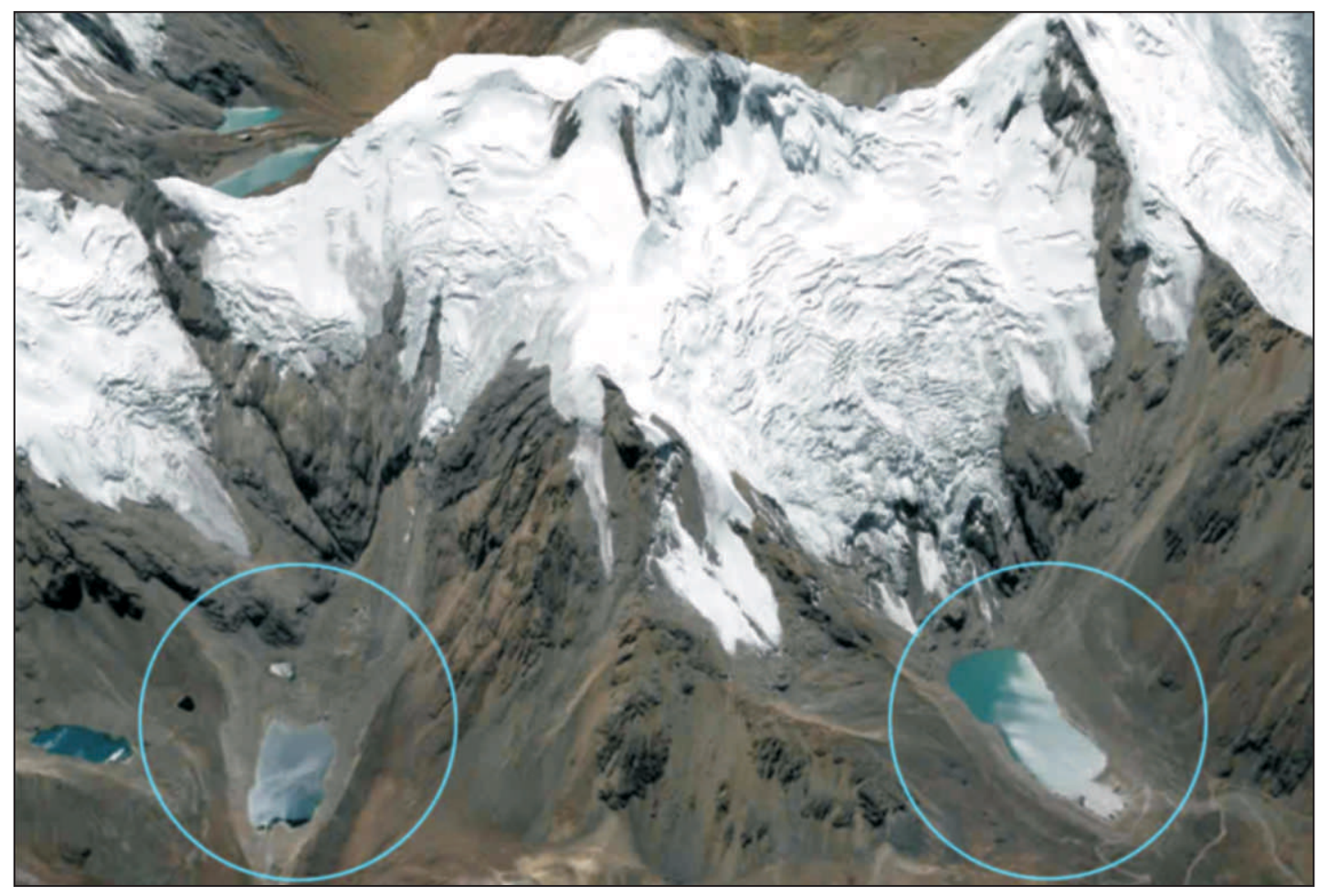

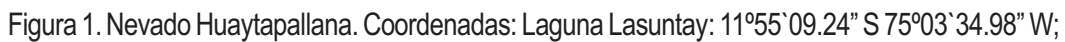
Laguna Chuspicocha 11054 $02.08^{\prime \prime} S 75^{\circ} 04^{\prime} 03.39^{\prime \prime} \mathrm{W}$

Fuente: Imagen de DigitalGlobe (Google earth 2011) 

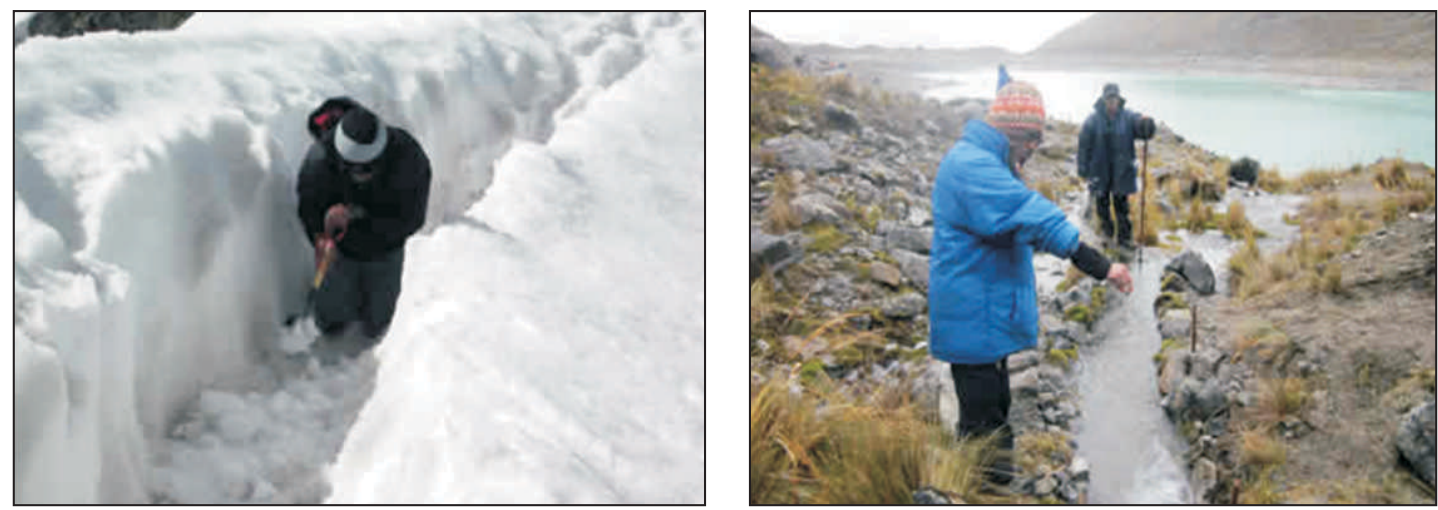

Figura 2, 3. Perforaciones y cálculo de caudal en la zona de estudio.

La ecuación básica del balance (b) de masa en un punto del glaciar para un instante, $\mathrm{db} / \mathrm{dt}$, se extrajo de la ecuación que planteó Lliboutry 1964, modificado por Paterson en 1994 y mejorado por Dyurgerov en 2002. (9)

$\frac{d b}{d t}=\rho \frac{d h}{d t}+\int \frac{d \rho}{d t} d y$

Donde $\rho$ es la densidad del hielo de espesor h que varía según el tiempo t. El primer término del lado derecho de la ecuación representa el cambio de masa de hielo (con densidad constante). El segundo término es el cambio de densidad de la columna de nieve de espesor y sobre el periodo de tiempo † (ecuación i). (10)

A partir de (ecuación i) y considerando dos instantes de medición, $t_{i} y t_{i}-1$, se obtiene el balance de masa para el instante i como:

$b_{i}=\rho_{o} \Delta h+\left(\rho y_{i}-\rho_{i-1} y_{i-1}\right)$

Considerando $\rho_{i}=\rho_{i-1}=\rho$, se tiene:

$b_{i}=\rho_{o}\left(h_{i}-h_{i-1}\right)+\rho\left(y_{i}-y_{i-1}\right)$

La toma de datos se llevó a cabo a través de tres etapas. La primera, corresponde a la división del glaciar en rangos de altura que fluctúa generalmente entre 25, 50 y 100 m; en la segunda etapa, se calcularon los valores medidos para cada rango. En algunos casos, debido a que no se realizaron ninguna perforación en rangos establecidos, los valores del balance se obtuvieron por interpolación. (11)
Finalmente, el balance neto específico $\mathrm{Bn}$, es el valor de balance ponderado por su superficie relativa utilizando la siguiente ecuación:

$B_{n}=\Sigma B_{i}(s i / S)$

Donde $B_{i}$ corresponde al balance de un rango de altura $\mathrm{i}, \mathrm{s}_{\mathrm{i}}$ la superficie del rango de altura y $S$ a la superficie total del glaciar.

\section{Balance hidrológico para el cálculo de escurrimiento:}

Finalmente a partir del método indirecto del balance hidrológico que consiste en establecer una comparación estacional entre la cantidad de hielo acumulado por precipitaciones sólidas medidas o estimadas (P), la ablación (evaporación y sublimación) medida o estimada (E) y la fusión (R) medida a poca distancia del glaciar, se obtuvo el balance hidrológico $\left(B_{h}\right)$ aplicando la ecuación siguiente para luego deducir el escurrimiento neto (12):

\section{$B_{h}=P-R-E$}

Sin tomar en cuenta la sublimación, pero considerando el promedio de las precipitaciones $(\mathrm{P})$, la superficie del glaciar $(S G)$, el caudal de derretimiento (D), la superficie total de la cuenca donde se localiza (S) y el coeficiente de escurrimiento $\left(c_{e}\right)$, el balance hidrológico $B_{h}$ se puede obtener de la siguiente manera: 
$B_{h}=P-1 / S G\left[D-(S-S G) C_{e} P\right]$

\section{RESULTADOS}

\section{Variaciones del régimen de acumu- lación y ablación de los nevados}

El régimen de acumulación ( $A C)$, observado en los glaciares llega hasta los $50 \mathrm{~mm}$ equivalente de agua entre los meses de diciembre a abril que corresponden al verano. En los meses de junio a octubre el régimen llega a niveles críticos de $-30 \mathrm{~mm}$ equivalente de agua. Este proceso se observa en las zonas de ablación (AB)

También se ha observado que en los meses de junio a noviembre que corresponde a la estación de invierno y parte de la primavera, ocurren los mayores torrentes de agua por una mayor aceleración en la fusión del nevado acumulado como se observa en la Figura $\mathrm{N}^{\circ} 4$.

\section{Efectos de las variaciones diurnas de la temperatura en la fusión de los nevados}

En la Figura $\mathrm{N}^{\circ} 5$, se muestra la evaluación los cambios en los caudales de los torrentes de agua en función del cambio de la temperatura durante el día. El proceso de cambio no guarda una estrecha relación con el aumento de temperatura, existe un desfase de seis horas con el nivel de caudal de torrentes de agua. Mientras que la temperatura llega a su máximo alrededor de las 12:00 m, el nivel máximo de caudal ocurre a las $06: 00 \mathrm{pm}$. El caudal mínimo de torrentes de agua si guarda una relación con la temperatura, ambos llegan a valores mínimos a las 03:00 am. La temperatura tiene un descenso de hasta $-5^{\circ} \mathrm{C}$ que influye en la solidificación del agua, mientras que a las 12:00 $\mathrm{m}$, la temperatura llega a valores de $6{ }^{\circ} \mathrm{C}$, acelerando el proceso de fusión que aumenta el caudal de los torrentes de agua.

Variación anual del régimen hídrico de los nevados

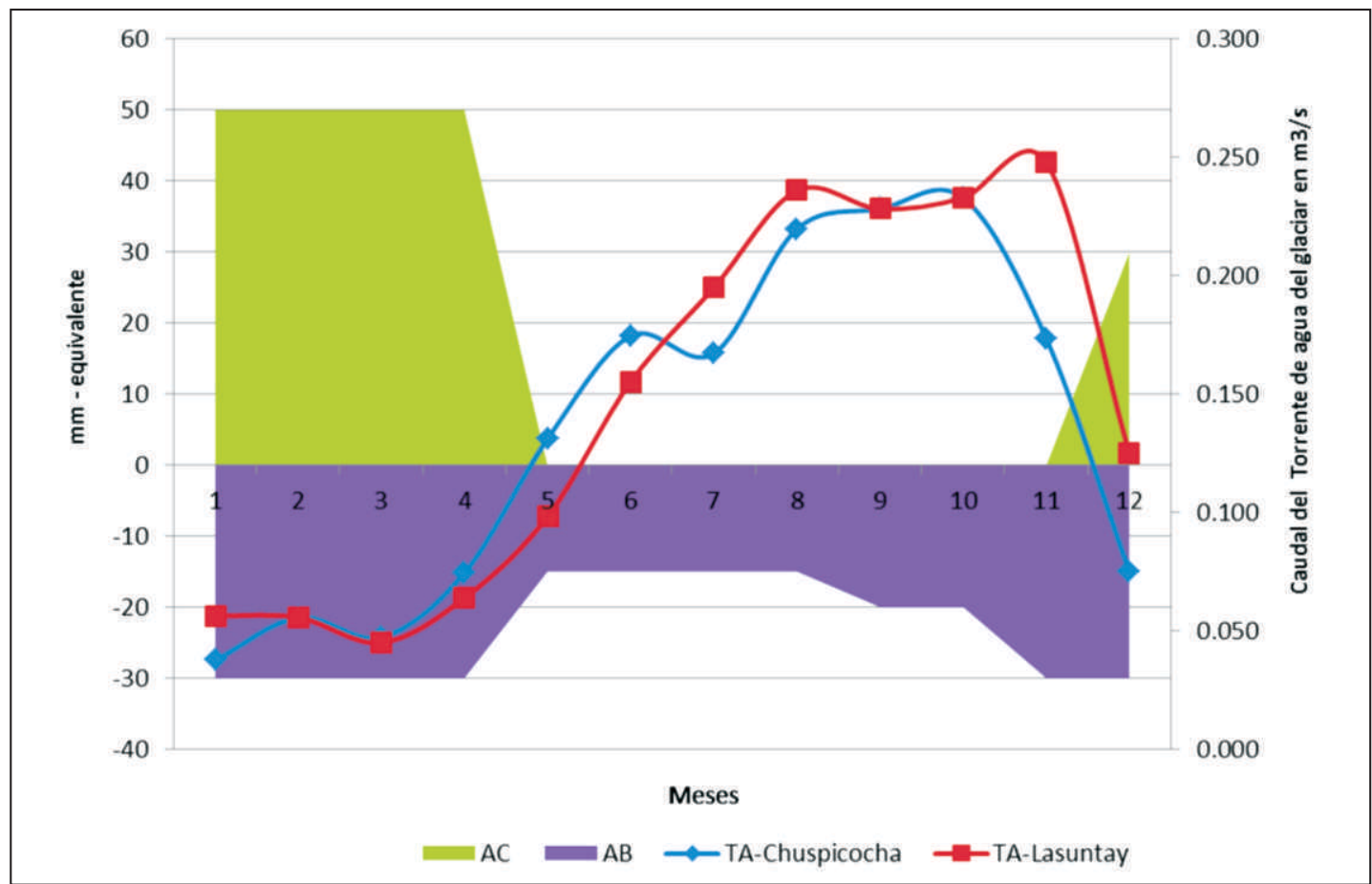

Figura 4. Régimen de acumulación y ablación con torrentes de agua del glaciar que emana a las lagunas de Chuspicocha y Lasuntay. 


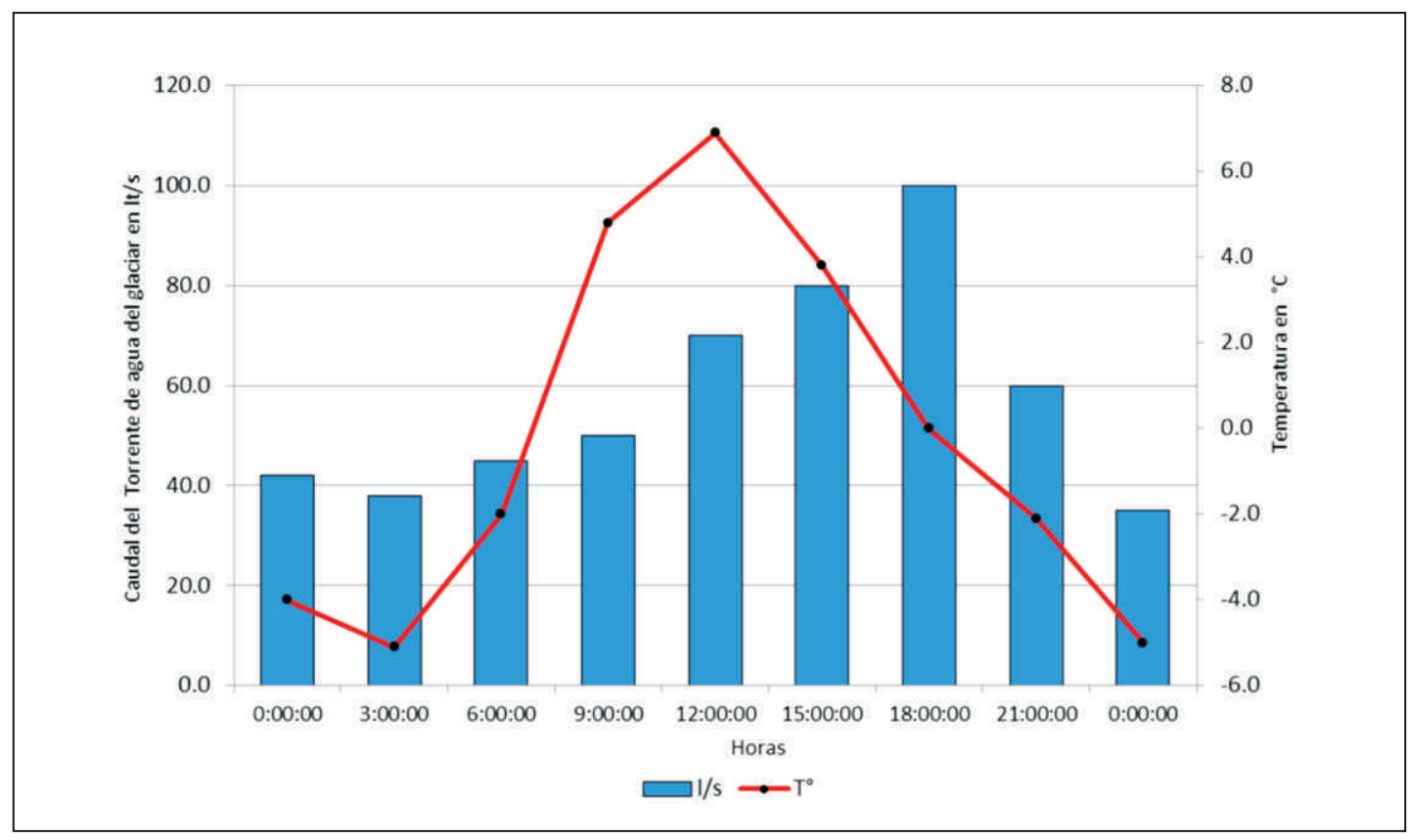

Figura 5. Variación de la Temperatura y del Torrente de agua del glaciar del Nevado del Huaytapallana.

La Figura 6, muestra la evaluación de la variación anual de los torrentes de agua que emanan a las lagunas desde las zonas de ablación de los glaciares del nevado del Huaytapallana. Los máximos caudales observados se presentan en los meses de agosto a noviembre para la laguna Lasuntay y desde junio a octubre para la laguna
Chuspicocha. Los niveles mínimos de torrentes de agua se observa en los meses de diciembre a abril.

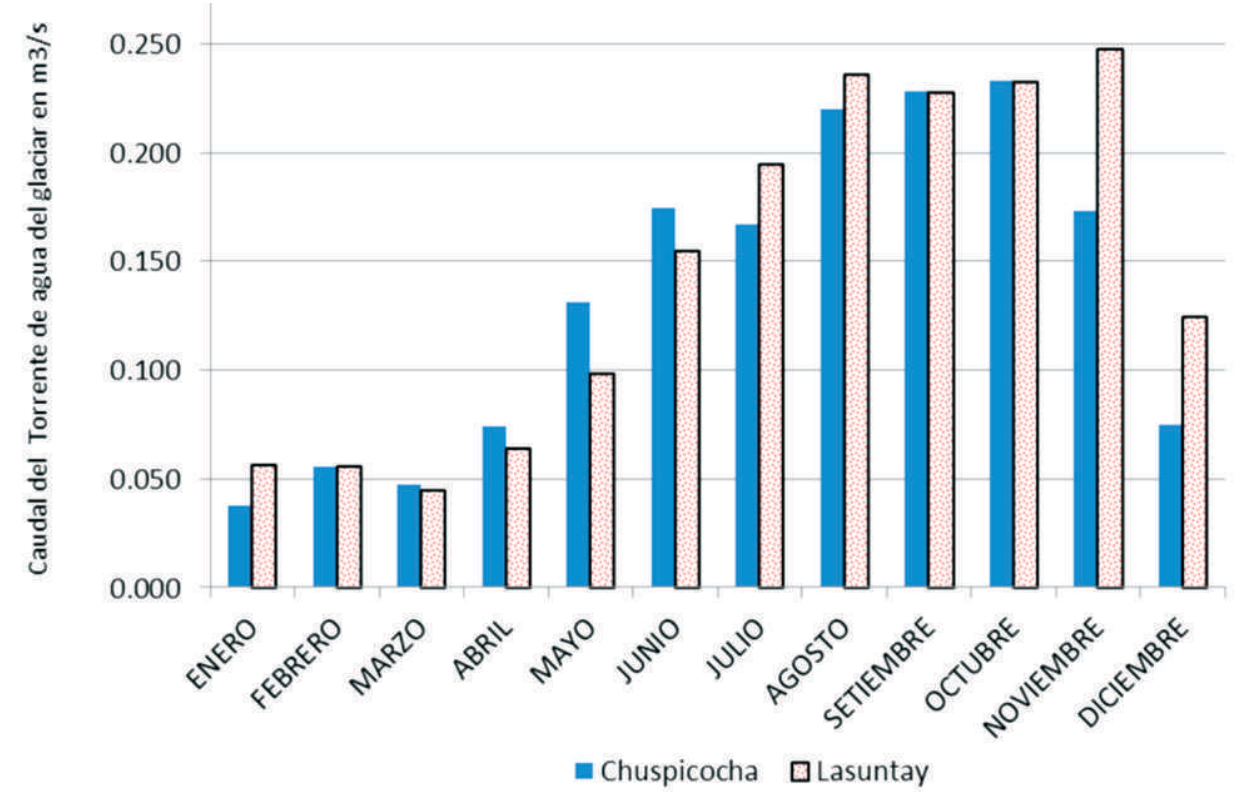

Figura 6.: Variación anual del régimen hídrico de caudales del nevado que escurren a las lagunas. 


\section{Régimen hídrico de la laguna Lasuntay y Chuspicocha durante estaciones del año hidrológico}

El régimen hídrico de las lagunas que se han evaluado pasan por variaciones estacionales en el nivel de agua acumulada, se ha observado que en el verano la acumulación neta llega a 1226700 m3; para el otoño presenta una disminución de 490680 m3; en el invierno disminuye a 245 $340 \mathrm{~m} 3$ con respecto a la estación del otoño y 736020 m3 con respecto a la estación de verano. Finalmente el estrés hídrico de ambas lagunas se presenta en la estación de primavera con $245340 \mathrm{~m} 3$, disminuyendo en $981360 \mathrm{~m} 3$ de agua con respecto a la acumulación alcanzada en la primavera como se puede observar en la Tabla $\mathrm{N}^{\circ} 01$ y la Figura $N^{\circ} 7$ y 8.

\section{Efectos de las precipitaciones en el nivel de acumulación del nevado}

La Figura $\mathrm{N}^{\circ}$ 9, muestra el efecto de las precipitaciones sólidas en el incremento de masa de acumulación de nieve durante el año. En los meses de diciembre a abril se retiene y acumula más nieve en los glaciares, siendo el mes de febrero y marzo los meses de máxima acumulación. Los meses de junio a noviembre son épocas de mínimas acumulaciones porque también las precipitaciones son escasas. Además estos meses corresponde al invierno en donde no existen precipitaciones y la humedad desciende.

La Figura $\mathrm{N}^{\circ} 10$, muestra la relación que tienen las precipitaciones y la acumulación de masas de nieve en los glaciares, existe una relación directa, indicando que es la temporada de precipitaciones del verano lluvioso que genera las acumulaciones máximas. También, se observa que existe un pequeño desfase entre las precipitaciones y el nivel de acumulación a partir del mes de setiembre, en donde las precipitaciones durante esta temporada no tienen mucha contribución en el sistema de acumulación por procesos de compensación.

Tabla Nº1: Régimen hídrico estacional de las lagunas Lasuntay y Chuspicocha.

\begin{tabular}{lcc} 
Estación & $\begin{array}{c}\text { Volumen acumulado } \\
\text { laguna Lasuntay } \\
\text { en } \mathrm{m}^{3}\end{array}$ & $\begin{array}{c}\text { Volumen acumulado } \\
\text { laguna Chuspicocha } \\
\text { en m3 }\end{array}$ \\
\hline Verano & 833400 & 393300 \\
Otoño & 500040 & 235980 \\
Invierno & 333360 & 157320 \\
Primavera & 166680 & 78660 \\
\hline
\end{tabular}
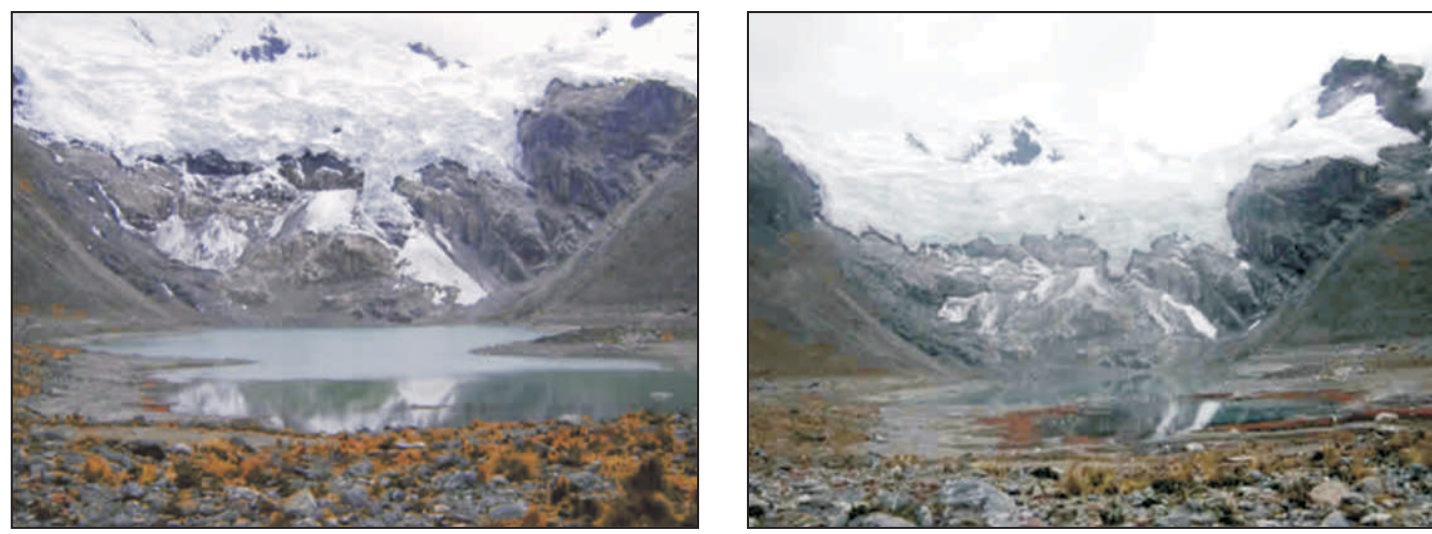

Figura 7, 8. Momentos de máxima y mínima acumulación de lámina de agua en las lagunas en la estación de verano e invierno. 


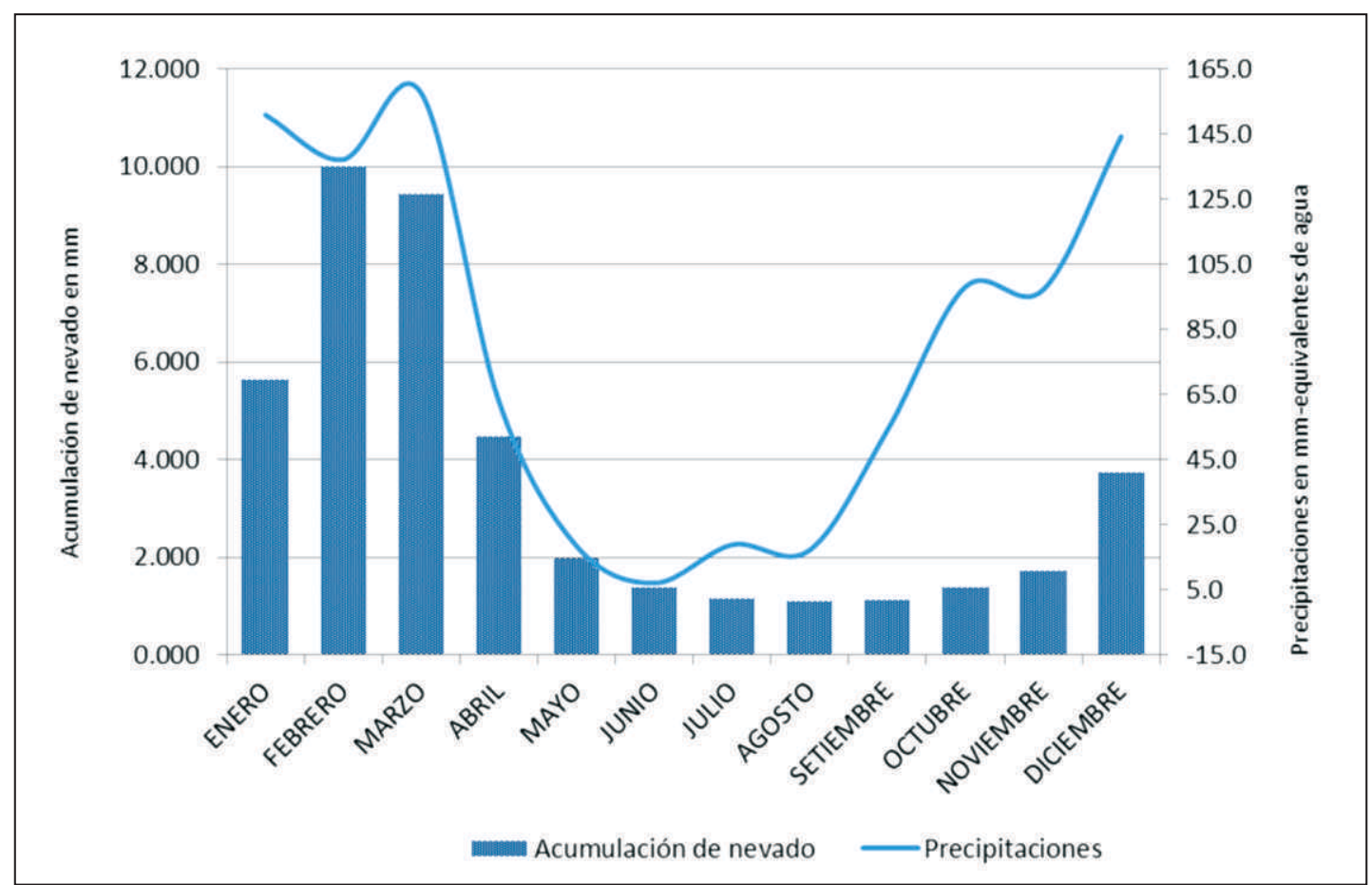

Figura 9: Caudal del río Shullcas y precipitaciones en forma líquida y sólida de las lagunas Chuspicocha y Lasuntay.

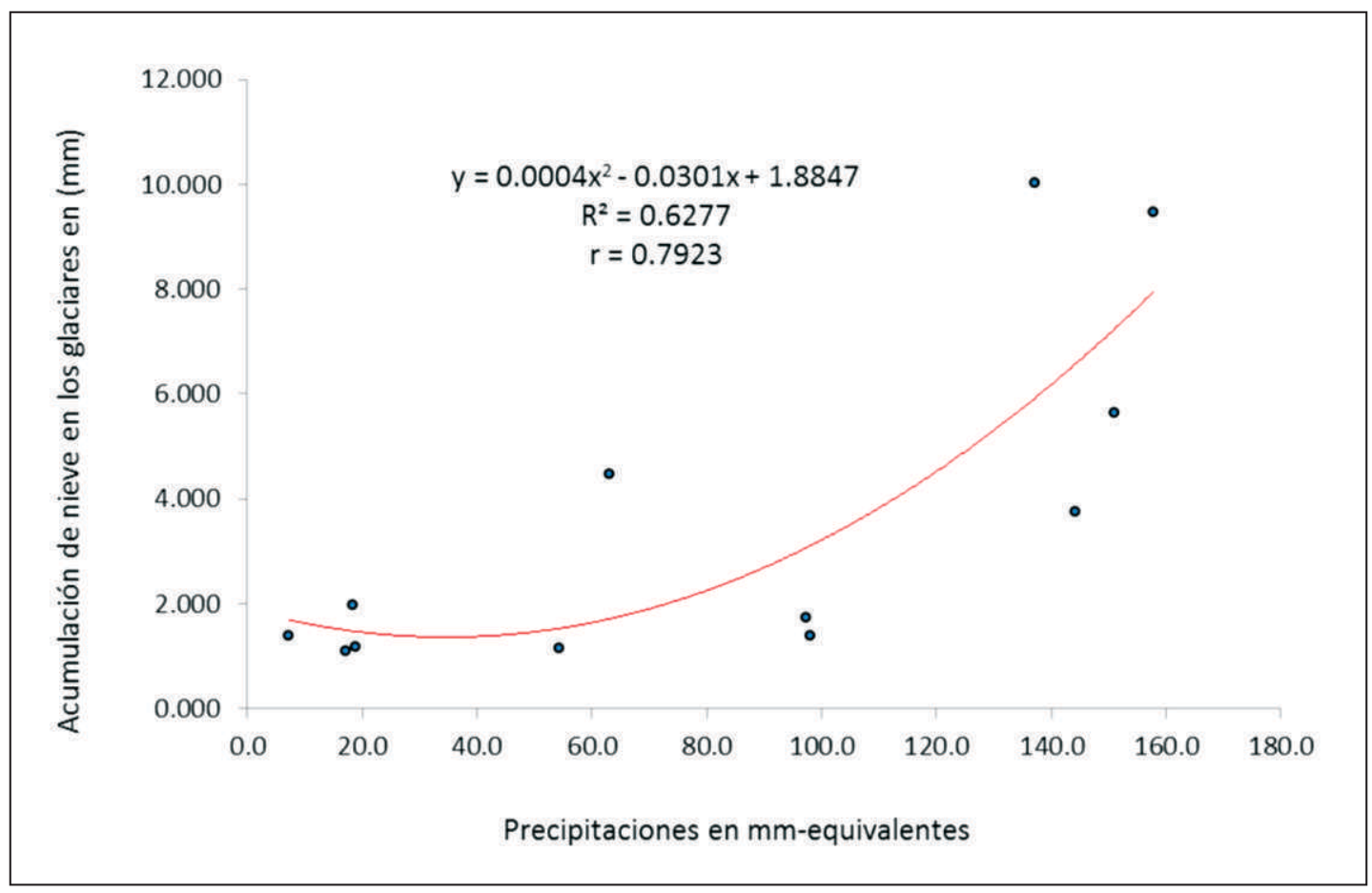

Figura 10. Análisis de correlación y regresión entre las precipitaciones y los niveles de acumulación de nieve. 


\section{DISCUSIÓN}

Durante el día el desfase que existe entre la temperatura y el volumen del caudal de los torrentes de agua que escuren a las lagunas, se debe al retardo de fusión que tiene la nieve acumulada, durante las primeras horas en que aumenta la temperatura, el proceso de descongelamiento demora por una pobre contribución calorífica a su masa, este mecanismo retarda el proceso en el tiempo, según Maisincho et. al. (1) al medio día cuando se tiene la más altas temperaturas, éstas no acelerarán el proceso de fusión pero si fusionan la mayor cantidad de masa de nieve, permitiendo que este mecanismo influya en el caudal máximo después de haber transcurrido 406 horas sobre el máximo de temperatura, Oerlemans y Fortuin. (9) Al respecto, en nuestro estudio se ha determinado que el proceso de cambio no guarda una estrecha relación con el aumento de temperatura, existe un desfase de seis horas con el nivel de caudal de torrentes de agua. Mientras que la temperatura llega a su máximo alrededor de las 12:00 m, el nivel máximo de caudal ocurre a las $06: 00 \mathrm{pm}$.

Kaser y Osmaston, mencionan que en la variación anual, el mayor flujo de torrentes de agua que escurren a la laguna Lasuntay y Chuspicocha, a partir de la fusión en la zona de ablación, ocurren en los meses que corresponden al invierno y primavera (8), coincidiendo con nuestros hallazgos, que se ha observado en los meses de junio a noviembre correspondiente a la estación de invierno y parte de la primavera, ocurren los mayores torrentes de agua por una mayor aceleración en la fusión del nevado.

El volumen de agua acumulado en las lagunas presentados en la Tabla 1, depende esencialmente del aporte de flujo de agua que recibe de los torrentes que emanan de los nevados por procesos de fusión y del aporte directo de las precipitaciones líquidas o sólidas sobre su superficie, Francou et. al. (7). Este proceso físico y atmosférico de aporte a la recarga hídrica, hace que los volúmenes máximos de acumulación de agua en las lagunas se obtenga en la estación de verano y parte del otoño, que corresponden a los meses de diciembre a abril, Figura $\mathrm{N}^{\circ}$ 06. A partir del cual el régimen de acumulación depende únicamente de los torrentes de agua que emanan de la fusión de los nevados del glaciar, Dyurgerov y Paterson. (5)

\section{Agradecimientos}

A Carlos Rau, Wyler Espeza, Milagros Carlos, Denisse Galarza, Miguel Ticse, Frans Cornejo y Edsel Arroyo por su apoyo técnico en los estudios de campo del glaciar. A CARE Perú y al proyecto PRAA por su apoyo incondicional en la investigación. 


\section{REFERENCIAS BIBLIOGRAFICAS}

1. Maisincho J, Mendoza J, Ramírez A, Soruco J, Taupin D, Wagnon P. Métodos de Observación de Glaciares en los Andes Tropicales. Mediciones de Terreno y Procesamiento de Datos: París: Ediciones IRD; 2004.

2. Francou B, Ribstein P, Wagnon P, Ramirez E, Pouyaud B. Glaciers of the tropical Andes: indicators of global climate variability. In: Huber U, Bugmann HKM, Reasoner MA. (Eds.), Global Change and Mountain Regions: An Overview of Current Knowledge, vol. 23. Springer, Dordrecht: Ediciones IRD; 2005.

3. Brasseur G. Physique et Chemique de l'Atmosphère moyenne, Masson. El Fin de las Cumbres Nevadas, Glaciares y Cambio Climático en la comunidad Andina, Paris: CAN, PNUMA, IRD, Agencia: Ediciones Española de Cooperación Internacional; 2007.

4. Baird D. An Introduction to Measurement Theory and Experiment Desig.. HJ: 3rd. ed. Prentice Hall, Englewood Cliffs; 1995.

5. Dyurgerov, M. Glacier Mass Balance and Regime: Data of Measurements and Analysis. Occasional Paper No. 55, Editors: Mark Meier (INSTAAR), Richard Armstrong (NSIDC), Institute of Arctic and Alpine Research, University of Colorado, Boulder; 2002.

6. Francou B, Ribstein P \& Saravia R. Monthy Balance and Water discharge on an intertropical glacier. The Zongo Glacier, Cordillera Real, Bolivia, 16 S. Journal of Glaciology; 1995.

7. Francou B, Vuille M, Wagnon P, Mendoza J \& Sicart JE. Tropical Cimate Change Recorded by a Glacier of the Central Andes During the Last Decades of the 20th century: Chacaltaya Bolivia, $16^{\circ}$ S. Journal of Geophysical Research; 2003.

8. Kaser G. Osmaston H. Tropical Glaciers. Cambridge: University Press - United Kingdom; 2002.

9. Oerlemans J, Fortuin J, Sensitivity of glaciers and small ice caps to Greenhouse warming, Berlin; 2002.

10. Ribstein P, Tiriau E, Francou B, et Saravia F. Tropical climate and glacier hydrology: A case study in Bolivia, Journal of Hydrology; 1995.

11.Pouyaud B, Francou P, Ribstein P. Un réseau d'observation des glaciers dans les Andes tropicales, Bulletin de l'institut français d'études andines; 1995.

12.Dyugerov J, Paterson V, Analysis of water of glaciers and snowfall caps to Greenhouse warming, Londres; 2006.

Correo electrónico:

jarroyox@gmail.com

pgurmendi@continental.edu.pe 